\title{
Effects of latitudinal distributions of particle density and wave power on cyclotron resonant diffusion rates of radiation belt electrons
}

\author{
Danny Summers and Binbin Ni \\ Department of Mathematics and Statistics, Memorial University of Newfoundland, St. John's, Newfoundland, Canada
}

(Received February 8, 2008; Revised March 25, 2008; Accepted March 26, 2008; Online published August 4, 2008)

\begin{abstract}
We evaluate cyclotron resonant interactions of radiation belt electrons with VLF chorus, plasmaspheric ELF hiss and electromagnetic ion cyclotron (EMIC) waves. We assume that the Earth's magnetic field is dipolar and that each wave mode has a Gaussian spectral density. The dependence of the resonant electron diffusion rates on the latitudinal distributions of particle density and wave power is examined. We find that while the diffusion rates can be sensitive to the latitudinal distributions of density and wave power, in general the sensitivity depends on wave mode, equatorial pitch-angle, electron energy and $L$-shell. We determine the effects of the latitudinal distributions of density and wave power on the electron precipitation loss timescale due to combined scattering by VLF chorus, ELF hiss and EMIC waves. Accurate modeling of radiation belt electron dynamics requires observational data on the global distributions of particle number density and wave power.
\end{abstract}

Key words: Earth's radiation belt, wave-particle interactions, magnetospheric plasma waves, electron precipitation.

\section{Introduction}

Electrons in the Earth's outer radiation belt $(3<L<7)$ undergo cyclotron resonant interactions with various modes of plasma wave including whistler-mode chorus, plasmaspheric hiss and electromagnetic ion cyclotron (EMIC) waves, e.g., see Summers et al. (2007a, b) and references therein. Whistler-mode (VLF) chorus waves are observed in the lower-density region outside the plasmasphere, typically in the frequency range $0.05-0.8 \Omega_{\mathrm{e}}$, where $\Omega_{\mathrm{e}}$ is the electron gyrofrequency (Meredith et al., 2001; Santolik et al., 2004). Hiss is a broadband whistler-mode ELF emission occurring inside the plasmasphere and drainage plumes in the frequency range $\sim 100 \mathrm{~Hz}-$ several $\mathrm{kHz}$ (Meredith et al., 2004). EMIC waves in the frequency range 0.1$5.0 \mathrm{~Hz}$ are observed in the plasmasphere, typically along the duskside plasmapause, and in drainage plumes (Fraser and Nguyen, 2001). Energy diffusion due to cyclotron resonance with VLF chorus is an effective mechanism for generating relativistic $(>1 \mathrm{MeV})$ electrons in the outer radiation belt during magnetic storms (Summers et al., 1998, 2002; Roth et al., 1999; Summers and Ma, 2000; Miyoshi et al., 2003; Horne et al., 2005; Omura and Summers, 2006). VLF chorus, ELF hiss, and EMIC waves can each cause pitch-angle scattering of electrons into the loss cone leading to precipitation losses from the outer zone (Summers and Thorne, 2003; Albert, 2003; Thorne et al., 2005).

Quasi-linear theory provides useful techniques for determining the average properties of cyclotron-resonant diffusion (e.g., Lyons, 1974; Summers, 2005; Albert, 2007). Summers et al. (2007a, b) calculate quasi-linear diffusion

Copyright (c) The Society of Geomagnetism and Earth, Planetary and Space Sciences (SGEPSS); The Seismological Society of Japan; The Volcanological Society of Japan; The Geodetic Society of Japan; The Japanese Society for Planetary Sciences; TERRAPUB. rates to determine timescales for radiation belt electron acceleration and loss due to cyclotron resonance with VLF chorus, ELF hiss and EMIC waves. Resonant diffusion rates depend on the assumed distributions of background particle density and wave power. The present investigation extends the work of Summers et al. (2007a, b) by examining the effects of latitudinal distributions of particle density and wave power on the resonant diffusion rates of radiation belt electrons for the aforementioned wave modes. In Section 2 we provide a short account of the quasi-linear theory required to calculate resonant diffusion rates. In Section 3 we present our calculations of the (bounce-averaged) electron diffusion rates for individual wave modes, and we also determine the effects of the latitudinal distributions of density and wave power on electron precipitation loss timescales due to combined scattering by VLF chorus, ELF hiss and EMIC waves. Finally, in Section 4 we summarize our results.

\section{Cyclotron Resonant Diffusion Rates}

We assume a homogeneous collisionless plasma immersed in a uniform background magnetic field in the presence of superposed electromagnetic waves. The relativistic quasi-linear diffusion equation for the gyrophase-averaged phase space density $\Phi$ is

$$
\begin{aligned}
\frac{\partial \Phi}{\partial t}= & \frac{1}{\sin \alpha} \frac{\partial}{\partial \alpha}\left(D_{\alpha \alpha} \sin \alpha \frac{\partial \Phi}{\partial \alpha}\right) \\
& +\frac{1}{\sin \alpha} \frac{\partial}{\partial \alpha}\left(D_{\alpha p} \sin \alpha \frac{\partial \Phi}{\partial p}\right) \\
& +\frac{1}{p^{2}} \frac{\partial}{\partial p}\left(p^{2} D_{p \alpha} \frac{\partial \Phi}{\partial \alpha}\right) \\
& +\frac{1}{p^{2}} \frac{\partial}{\partial p}\left(p^{2} D_{p p} \frac{\partial \Phi}{\partial p}\right),
\end{aligned}
$$


where $D_{\alpha \alpha}, D_{\alpha p}=D_{p \alpha}$, and $D_{p p}$ are the cyclotron resonant diffusion coefficients which depend on the properties of the waves; $p=\gamma m v$ is the particle momentum where $v$ is the particle speed and $m$ is the rest mass; $\gamma=\left(1-v^{2} / c^{2}\right)^{-1 / 2}$ is the Lorentz factor $(c$ is the speed of light); $\alpha$ is the pitch-angle, and $t$ denotes time. The diffusion coefficients,

$$
\begin{aligned}
& D_{\alpha \alpha}=\overline{(\Delta \alpha)^{2}} /(2 \Delta t), \\
& D_{\alpha p}=\overline{(\Delta \alpha)(\Delta p)} /(2 \Delta t), \\
& D_{p p}=\overline{(\Delta p)^{2}} /(2 \Delta t)
\end{aligned}
$$

are determined from the ensemble-averaged particle-orbit corrections. Explicit formulae for $D_{\alpha \alpha}, D_{\alpha p}, D_{p p}$ corresponding to field-aligned (R-mode and L-mode) electromagnetic waves have been derived by Summers (2005) and Summers et al. (2007a). We assume that the waves have a Gaussian spectral density of the form,

$$
\tilde{W}(\omega)=\frac{(\Delta B)^{2}}{8 \pi} \frac{1}{\rho} \frac{1}{\delta \omega} e^{-\left(\frac{\omega-\omega_{\mathrm{m}}}{\delta \omega}\right)^{2}},
$$

with

$$
\rho=\frac{\sqrt{\pi}}{2}\left[\operatorname{erf}\left(\frac{\omega_{\mathrm{m}}-\omega_{1}}{\delta \omega}\right)+\operatorname{erf}\left(\frac{\omega_{2}-\omega_{\mathrm{m}}}{\delta \omega}\right)\right],
$$

where $\omega$ is the wave frequency, $\omega_{1}$ is the lower frequency limit, $\omega_{2}$ is the upper frequency limit, $\omega_{\mathrm{m}}$ is the frequency of maximum wave power, $\delta \omega$ is a measure of the bandwidth, and erf is the error function. The wave spectral density (3) has been normalized so that

$$
\frac{(\Delta B)^{2}}{8 \pi}=\int_{\omega_{1}}^{\omega_{2}} \tilde{W}(\omega) d \omega
$$

where $\Delta B$ is the mean wave amplitude.

In the present study we consider electron interaction with (R-mode) VLF chorus, (R-mode) ELF hiss, and (L-mode) EMIC waves. We assume a hydrogen plasma and we consider only field-aligned waves. Under the conditions of field-aligned wave propagation, all cyclotron harmonics $n$ are omitted except $n=-1$ and $n=+1$ which correspond respectively to R-mode and L-mode waves. In many cases first-order-harmonic diffusion rates provide a good approximation to diffusion rates for oblique waves calculated using higher-order resonances. For a given wave mode, the local diffusion coefficients can be expressed as functions of particle kinetic energy, $E=E_{\mathrm{k}} /\left(m c^{2}\right)=\gamma-1$, and pitch-angle $\alpha$. The diffusion coefficients depend further on the electron gyrofrequency $\Omega_{\mathrm{e}}$, the cold-plasma parameter $\alpha^{*}=\Omega_{\mathrm{e}}^{2} / \omega_{\mathrm{pe}}^{2}$ where $\omega_{\mathrm{pe}}$ is the electron plasma frequency, and the wave parameters $\omega_{1}, \omega_{2}, \omega_{\mathrm{m}}, \delta \omega$, and $\Delta B$ (Summers, 2005; Summers et al., 2007a). In order to calculate diffusion rates in a magnetic mirror geometry such as the Earth's magnetic field, the local diffusion coefficients $D_{\alpha \alpha}, D_{\alpha p}, D_{p p}$ must be bounce-averaged, i.e., averaged over particle bounce orbits. Summers et al. (2007a) have carried out this procedure assuming a dipole magnetic field to obtain the bounce-averaged diffusion coefficients $\left\langle D_{\alpha \alpha}\right\rangle$, $\left\langle D_{\alpha p}\right\rangle,\left\langle D_{p p}\right\rangle$ as functions of the kinetic energy $E$ and the equatorial pitch-angle $\alpha_{\mathrm{eq}}$ of the particle. In the following section we utilize the bounce-averaged diffusion coefficients given by Summers et al. (2007a) to determine how diffusion rates for radiation belt electrons depend on the latitudinal distributions of particle density and wave power.

\section{Results}

\subsection{Electron interaction with whistler-mode chorus}

Whistler-mode chorus comprises short $\left(\sim 10^{-1} \mathrm{sec}\right)$ discrete emissions that are quasi-monochromatic (e.g., Santolik et al., 2004). Electron interaction with such narrowband phase-coherent emissions cannot strictly be treated by quasi-linear theory. Nevertheless, herein we average over a specified band of chorus, and we assume that chorus can be represented by a weakly turbulent continuous spectrum. Accordingly, quasi-linear theory can be expected to provide an overall description of cyclotron resonant diffusion, though a quasi-linear treatment cannot take account of nonlinear effects such as phase trapping by the wave field.

In Fig. 1 we plot the bounce-averaged pitch-angle diffusion rate $\left\langle D_{\alpha \alpha}\right\rangle$, mixed (pitch-angle/momentum ) diffusion rate $\left\langle\left|D_{\alpha p}\right|\right\rangle / p$ and momentum diffusion rate $\left\langle D_{p p}\right\rangle / p^{2}$ for whistler-mode chorus for electron energies 100, 200, 500, $1000 \mathrm{keV}$ at $L=4$. We adopt a Gaussian wave spectral density with $\omega_{1}=0.05 \Omega_{\mathrm{e}}, \omega_{2}=0.65 \Omega_{\mathrm{e}}, \omega_{\mathrm{m}}=0.35 \Omega_{\mathrm{e}}$, $\delta \omega=0.15 \Omega_{\mathrm{e}}, \Delta B=100 \mathrm{pT}$, and we assume that the wave amplitude is constant along the field line. We compare the case of particle density $N(\lambda)=$ constant $=N_{\text {eq }}$ with that in which $N$ varies as the dipole magnetic field strength, namely, $N(\lambda) / N_{\mathrm{eq}}=B(\lambda) / B_{\mathrm{eq}}=f(\lambda)=$
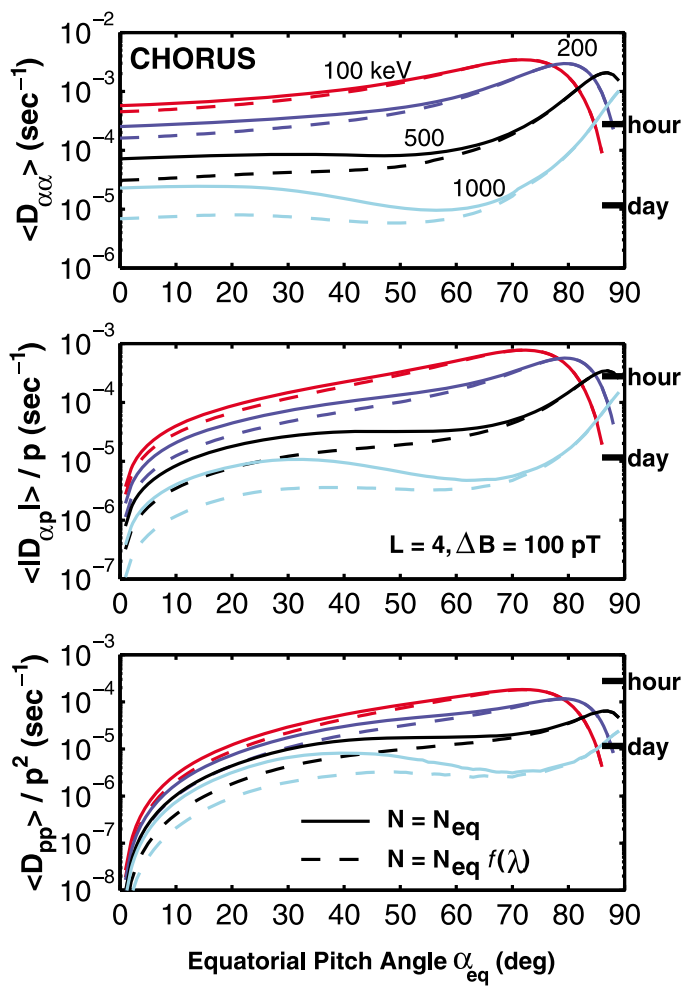

Fig. 1. Bounce-averaged diffusion coefficients for whistler-mode chorus for the indicated electron energies at $L=4$. Two latitudinal distributions of particle density are specified: (a) $N(\lambda)=N_{\text {eq }}=$ constant, (b) $N(\lambda)=N_{\text {eq }} f(\lambda)$ where $f(\lambda)=\left(1+3 \sin ^{2} \lambda\right)^{1 / 2} / \cos ^{6} \lambda$. 
(a) $E_{k}=200 \mathrm{keV}$
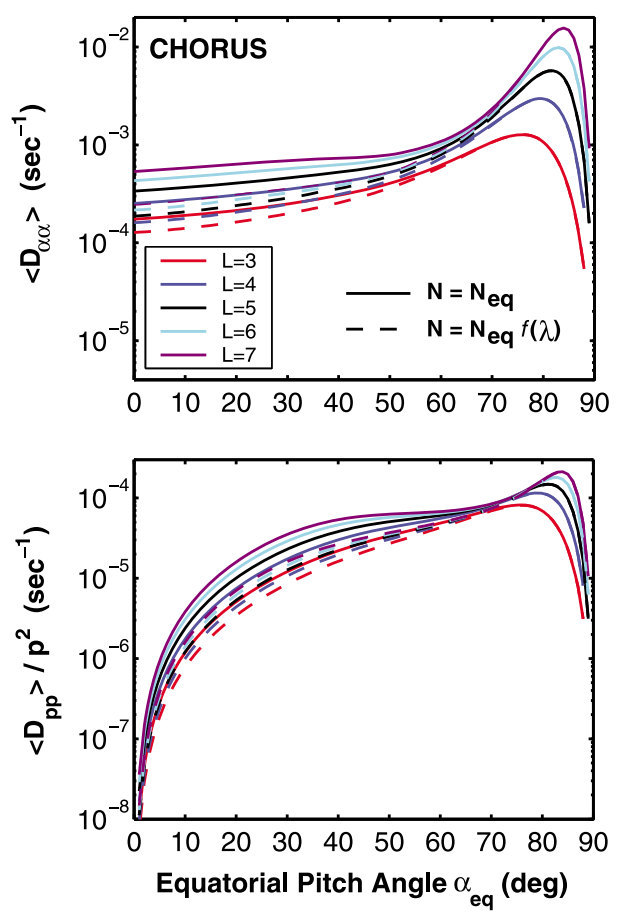

(b) $E_{k}=1 \mathrm{MeV}$
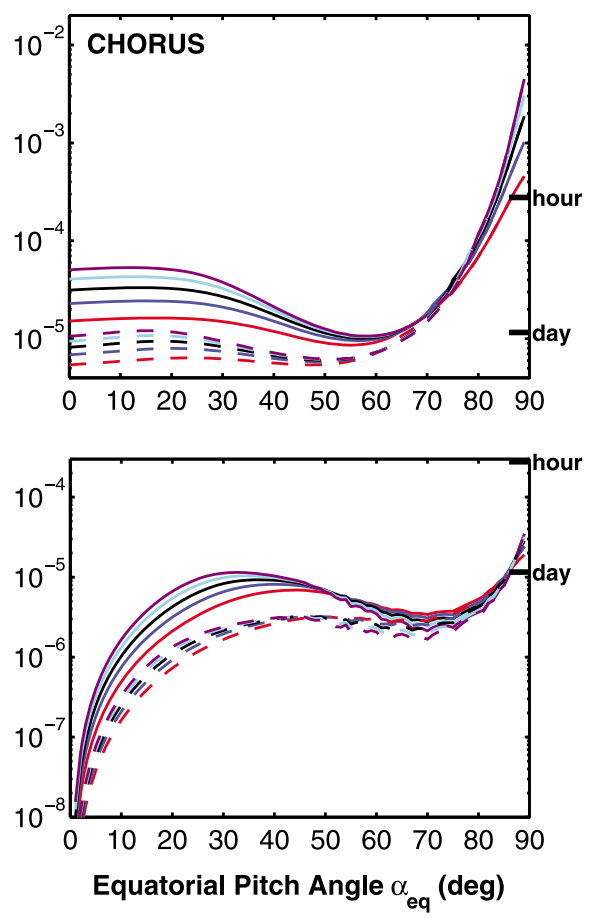

Fig. 2. Bounce-averaged pitch-angle diffusion rates (top) and momentum diffusion rates (bottom) for whistler-mode chorus for the indicated electron energies and $L$-values. The two chosen latitudinal distributions of particle density are as specified in Fig. 1.

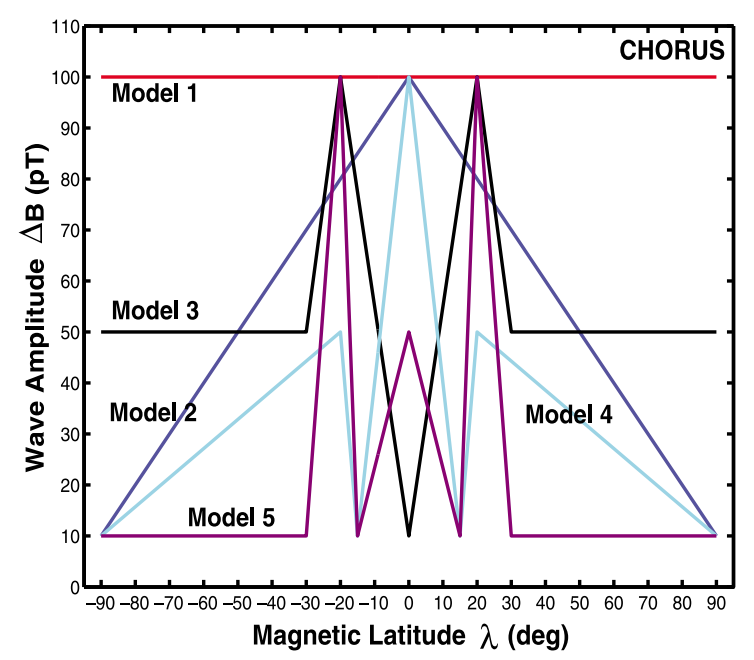

Fig. 3. Five models for the latitudinal distribution of chorus wave amplitude.

$\left(1+3 \sin ^{2} \lambda\right)^{1 / 2} / \cos ^{6} \lambda$ where $\lambda$ is the magnetic latitude. We take $N_{\text {eq }}=39 \mathrm{~cm}^{-3}$ and $\alpha_{\mathrm{eq}}^{*}=\left(\Omega_{\mathrm{e}}^{2} / \omega_{\mathrm{pe}}^{2}\right)_{\mathrm{eq}}=0.058$ at $L=4$. The effect of increasing particle density with latitude changes all three diffusion rates, the magnitude of the change depending on equatorial pitch-angle and electron kinetic energy. Larger changes occur at lower equatorial pitch-angles. This is because electrons with small values of $\alpha_{\mathrm{eq}}$ bounce to higher latitudes where the density increases substantially in the case $N(\lambda) \propto f(\lambda)$. At all energies, an increase in particle density with latitude reduces the pitchangle scattering rates near the loss cone but barely affects momentum diffusion rates for electrons with high equato-
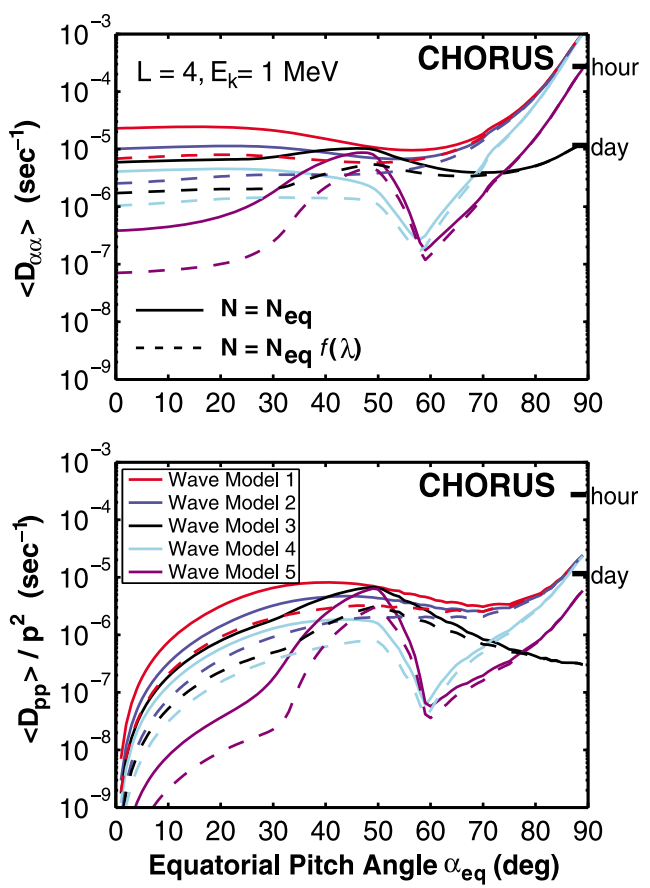

Fig. 4. Bounce-averaged pitch-angle diffusion rates (top) and momentum diffusion rates (bottom) for whistler-mode chorus for $1 \mathrm{MeV}$ electrons at $L=4$. The two chosen latitudinal distributions of particle density are as specified in Fig. 1, and the five wave models are as specified in Fig. 3.

rial pitch-angles $\left(70^{\circ}<\alpha_{\mathrm{eq}}<90^{\circ}\right)$. Thus, an increasing latitudinal number density increases the loss timescales for electrons of all energies, but has little effect on acceleration of electrons mirroring at lower latitudes. 

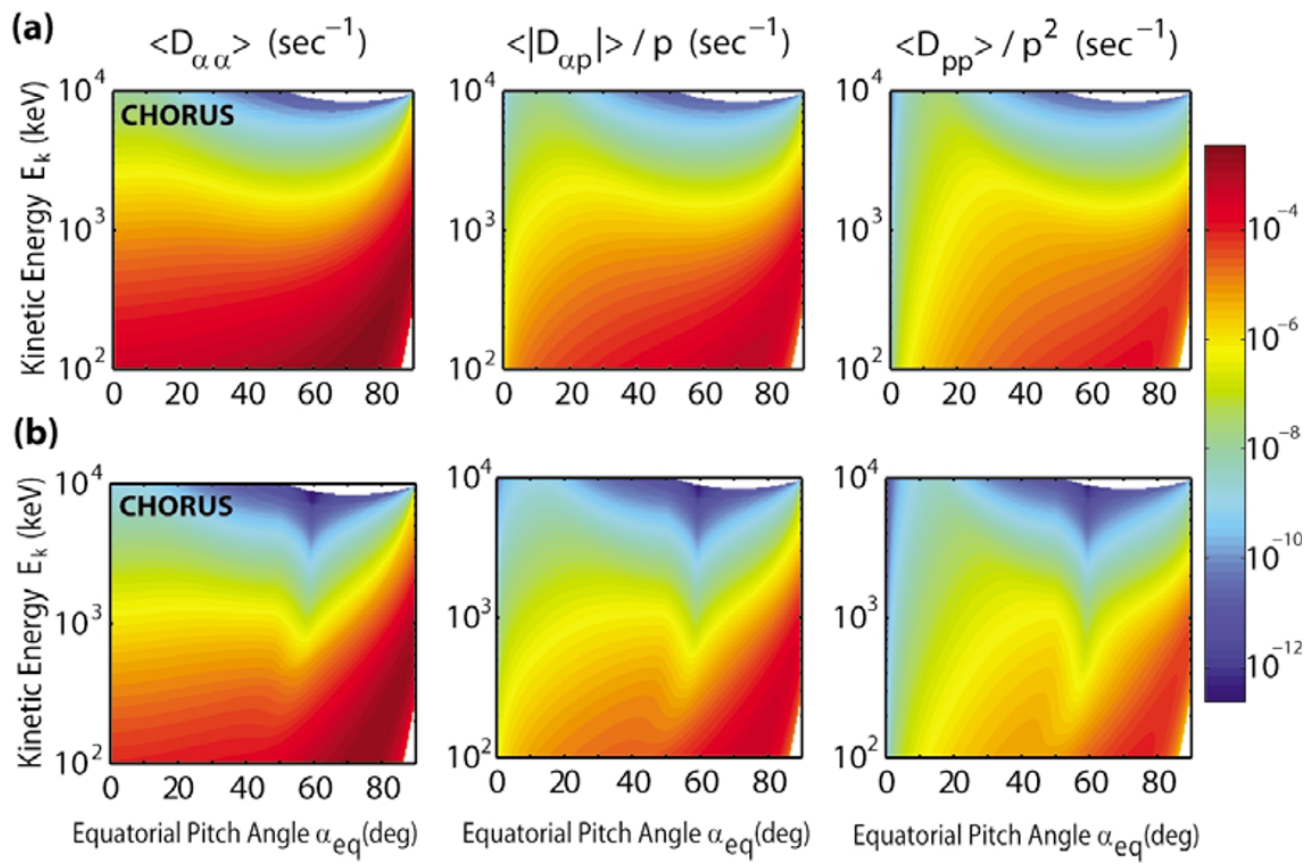

Fig. 5. Two-dimensional plots of bounce-averaged diffusion coefficients for whistler-mode chorus at $L=4$ as functions of equatorial pitch-angle and kinetic energy. The assumed latitudinal density distribution is $N(\lambda)=N_{\text {eq }} f(\lambda)$ where $f(\lambda)=\left(1+3 \sin ^{2} \lambda\right)^{1 / 2} / \cos ^{6} \lambda$. For the latitudinal distribution of chorus we adopt Wave Model 1 in the top panels and Wave Model 4 in the bottom panels (see Fig. 3).

In Fig. 2 for the same latitudinal distributions of particle number density used in Fig. 1 we calculate the pitch-angle and momentum diffusion rates for whistler-mode chorus for electron energies $200 \mathrm{keV}, 1 \mathrm{MeV}$ at a specified range of $L$ shells. We adopt the same chorus wave parameters used in Fig. 1 and we assume that the wave amplitude is constant along field lines. We use the equatorial "trough" particle density model $N_{\text {eq }}=124(3 / L)^{4} \mathrm{~cm}^{-3}$ due to Sheeley et al. (2001). Figure 2 shows generally that as $L$ decreases, for both $200 \mathrm{keV}$ and $1 \mathrm{MeV}$ electrons, diffusion rates decrease. As an exception, for $1 \mathrm{MeV}$ electrons momentum diffusion rates are largely independent of $L$-shell at large values of equatorial pitch-angle $\left(70^{\circ}<\alpha_{\text {eq }}<90^{\circ}\right)$, as was also found by Summers et al. (2007b).

In a study of the substorm dependence of chorus wave amplitudes Meredith et al. (2001) identifies equatorial $\left(|\lambda|<15^{\circ}\right)$ and high-latitude $\left(15^{\circ}<|\lambda|<90^{\circ}\right)$ chorus emissions outside the plasmasphere. We choose a set of five models of latitudinal distribution of wave amplitude to represent equatorial and high-latitude source strengths of chorus emissions of differing magnitudes. These models are illustrated in Fig. 3. For Model $1, \Delta B(\lambda)=$ constant $=100$ pT. Models 2 and 3 respectively represent an equatorial source only and a high-latitude source only. Models 4 and 5 include both equatorial and high-latitude sources, but with different relative strengths. In Fig. 4 we plot bounce-averaged pitch-angle diffusion rates (top) and momentum diffusion rates (bottom) for whistler-mode chorus for $1 \mathrm{MeV}$ electrons at $L=4$. We adopt the same chorus wave parameters and latitudinal density distributions as used in Fig. 1, and we calculate the diffusion rates for each of the wave models given in Fig. 3. We see from Fig. 4 that the diffusion rates are influenced by the high-latitude wave distributions for small equatorial pitch-angles, and by the low-latitude wave distributions for large equatorial pitch-angles. For small (large) equatorial pitch-angles the diffusion rates increase as the overall strength of the highlatitude (low-latitude) wave distribution increases. In Fig. 4 the dependence of the diffusion rates on the latitudinal density distributions is similar to that found in Figs. 1 and 2.

In Fig. 5 we provide two-dimensional plots of the bounce-averaged diffusion coefficients for whistler-mode chorus at $L=4$, versus equatorial pitch-angle $\alpha_{\mathrm{eq}}$ and kinetic energy $E_{\mathrm{k}}$. We assume the density distribution $N(\lambda)=N_{\text {eq }} f(\lambda)$, and for the waves we adopt Model 1 in the top panels and Model 4 in the bottom panels. Figure 5 shows how a change in the latitudinal wave distribution can produce significant changes in the diffusion coefficients over broad regions of $\left(\alpha_{\mathrm{eq}}, E_{\mathrm{k}}\right)$-space.

\subsection{Electron interaction with plasmaspheric hiss}

Reinisch et al. (2001) and Denton et al. (2006) have carried out experimental studies of the plasma density distribution along field lines in the plasmasphere. Motivated by such studies, we adopt the functional forms $N(\lambda)=$ $N_{\text {eq }}[f(\lambda)]^{\sigma}$ where $\sigma=0,0.6,1,1.5$, as representative of a realistic range of latitudinal density distributions in the plasmasphere at $L=4$. We plot the electron number density profiles corresponding to these four models in Fig. 6. In the top and middle panels of Fig. 7 we present bounceaveraged pitch-angle diffusion rates for plasmaspheric hiss for electron energies $200 \mathrm{keV}, 500 \mathrm{keV}$, and $1 \mathrm{MeV}$ at $L=4$. A Gaussian wave spectral density is assumed with $\omega_{1} / 2 \pi=100 \mathrm{~Hz}, \omega_{2} / 2 \pi=2000 \mathrm{~Hz}, \omega_{\mathrm{m}} / 2 \pi=550 \mathrm{~Hz}$, $\delta \omega / 2 \pi=300 \mathrm{~Hz}$. We set $N_{\mathrm{eq}}=346 \mathrm{~cm}^{-3}$ and $\alpha_{\mathrm{eq}}^{*}=$ 0.0066. In the top panel we put $\Delta B=40 \mathrm{pT},|\lambda|<15^{\circ}$; $\Delta B=50 \mathrm{pT}, 15^{\circ}<|\lambda|<90^{\circ}$ (Meredith et al., 2004), and we use the four latitudinal density distributions shown in Fig. 6. In the middle panel we put $N(\lambda)=N_{\text {eq }}=$ con- 


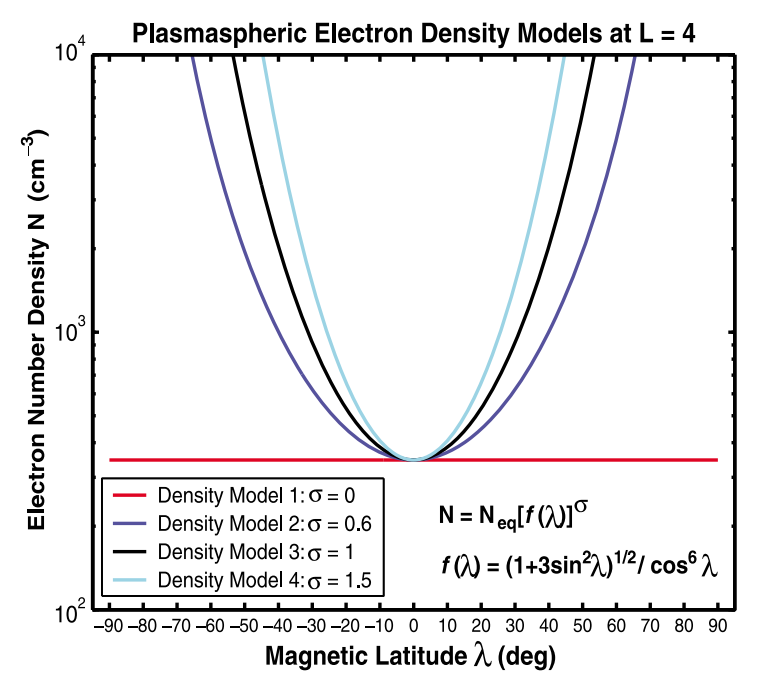

Fig. 6. Four models for the latitudinal distribution of plasmaspheric electron density at $L=4$.

stant, and we specify four latitudinal distributions of hiss wave power. The wave models comprise Wave Model I: $\left[\Delta B=10 \mathrm{pT},|\lambda|<90^{\circ}\right.$ ]; Wave Model II: $[\Delta B=40 \mathrm{pT}$, $|\lambda|<15^{\circ} ; \Delta B=50 \mathrm{pT}, 15^{\circ}<|\lambda|<30^{\circ} ; \Delta B=0$, $30^{\circ}<|\lambda|<90^{\circ}$ ]; Wave Model III: $[\Delta B=40 \mathrm{pT}$, $|\lambda|<15^{\circ} ; \Delta B=50 \mathrm{pT}, 15^{\circ}<|\lambda|<90^{\circ}$ ]; Wave Model IV: $\left[\Delta B=100 \mathrm{pT},|\lambda|<90^{\circ}\right]$. Wave Models I-IV represent a range of hiss distributions from weak (I) to relatively strong (IV). No drift-averaging is carried out in Fig. 7. In the top panel of Fig. 7 we see that as the density distributions become stronger, diffusion rates become weaker at lower equatorial pitch-angles, for all energies, while at higher equatorial pitch-angles diffusion rates are largely independent of density distribution (as we found in Fig. 1 for chorus). Similarly, in the middle panel of Fig. 7, as the wave distributions become stronger the diffusion rates increase over a wide range of equatorial pitch-angles except those toward $90^{\circ}$. We note also in Fig. 7 (middle panel) that there is no distinction between the diffusion rates at energies $200 \mathrm{keV}$ and $500 \mathrm{keV}$ for the Wave Models II and III, whereas such a distinction exists at $1 \mathrm{MeV}$. The explanation is that $\mathrm{MeV}$ electrons are in resonance at higher latitudes $\left(|\lambda|>30^{\circ}\right)$ whereas $200 \mathrm{keV}, 500 \mathrm{keV}$ electrons are not. Thus, the difference between Wave Models II and III is irrelevant for lower energy electrons.

In the bottom panel of Fig. 7 we show a two-dimensional plot of the bounce-averaged pitch-angle diffusion rate for hiss at $L=4$, as a function of equatorial pitch-angle and kinetic energy. For this plot we adopt Wave Model III, which represents a fairly strong hiss distribution, and density model 2 with $\sigma=0.6$ (see Fig. 6).

\subsection{Electron interaction with EMIC waves}

In the top and middle panels of Fig. 8 we present bounceaveraged pitch-angle diffusion rates for EMIC waves for electron energies $2.1 \mathrm{MeV}, 3 \mathrm{MeV}, 5 \mathrm{MeV}, 10 \mathrm{MeV}$ at $L=4$. A Gaussian wave spectral density is assumed with $\omega_{1}=\Omega_{\mathrm{p}} / 6, \omega_{2}=\Omega_{\mathrm{p}} / 2, \omega_{\mathrm{m}}=\Omega_{\mathrm{p}} / 3, \delta \omega=\Omega_{\mathrm{p}} / 6$ (where $\Omega_{\mathrm{p}}$ is the proton gyrofrequency), and $\Delta B=1 \mathrm{nT}$. We set $N_{\text {eq }}=346 \mathrm{~cm}^{-3}$ and $\alpha_{\text {eq }}^{*}=0.0066$. In the top panel
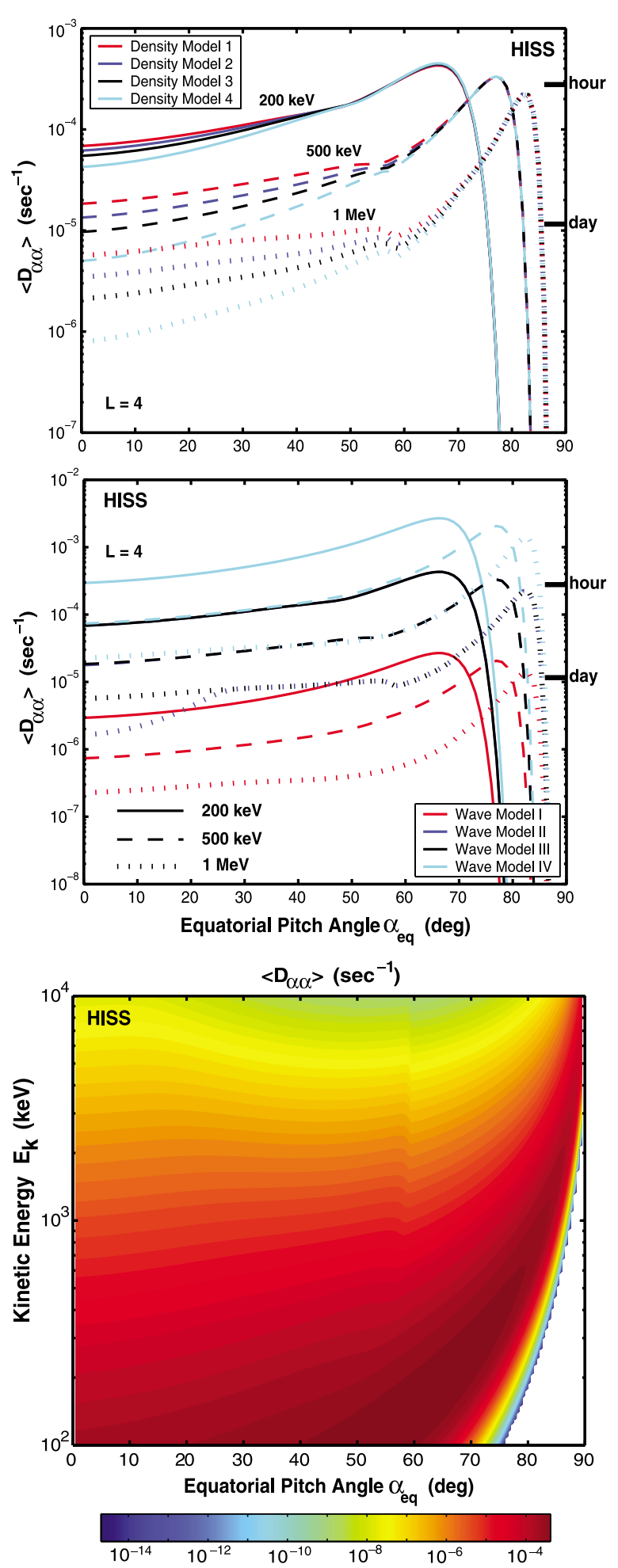

Fig. 7. In the top and middle panels, bounce-averaged pitch-angle diffusion rates for whistler-mode hiss for the indicated electron energies at $L=4$. The plasmaspheric density models are as specified in Fig. 6 . The four wave models are specified in the text. In the bottom panel, two-dimensional plot of the bounce-averaged pitch-angle diffusion rate for hiss at $L=4$, corresponding to Wave Model III and Density Model 2 .

we assume the wave amplitude is constant along the field line and we use the four latitudinal density distributions shown in Fig. 6. In the middle panel we put $N(\lambda)=N_{\text {eq }}=$ constant and we adopt the five latitudinal distributions of 

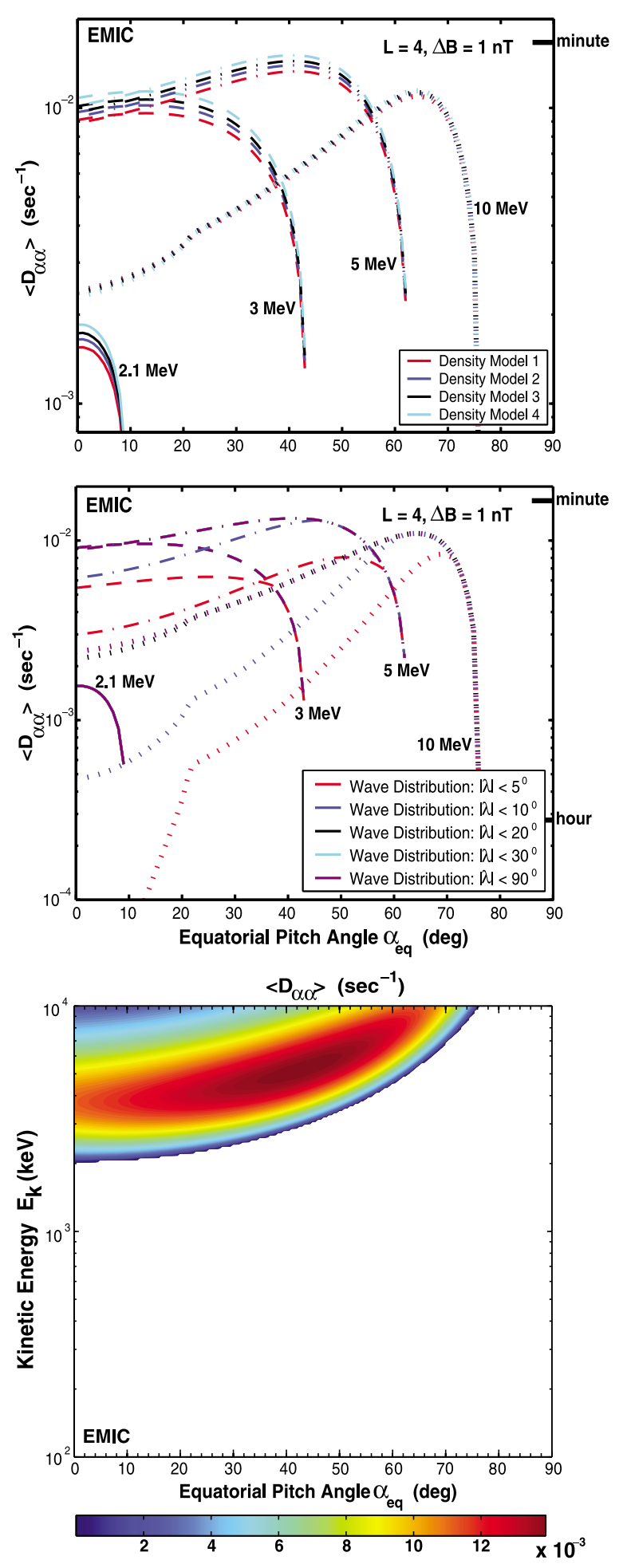

Fig. 8. In the top and middle panels, bounce-averaged pitch-angle diffusion rates for EMIC waves for the indicated electron energies at $L=4$. The plasmaspheric density models are as specified in Fig. 6. In the bottom panel, two-dimensional plot of the bounce-averaged pitch-angle diffusion rate for EMIC waves at $L=4$, corresponding to Wave Distribution $|\lambda|<20^{\circ}$ and Density Model 2.

wave power indicated in the figure. No drift-averaging is carried out in Fig. 8. In Fig. 8 (top) for electron energies $2.1 \mathrm{MeV}, 3 \mathrm{MeV}, 5 \mathrm{MeV}$, diffusion rates increase as the density distribution becomes stronger (in contrast to the hiss diffusion rates in Fig. 7 (top)). At such energies EMIC wave-electron resonance occurs relatively close to the equator; a density increase then increases the region of resonance causing a corresponding increase in diffusion rate. However, for $10 \mathrm{MeV}$ electrons, whose resonance region extends to higher latitudes, there is little distinction in the diffusion rate curves as the density distribution changes. For wave-particle interactions, in general, an increase in particle density causes a decrease in diffusion rate at any point. However, an increase in density may increase the latitudinal region of resonance and hence increase the diffusion rate. Whether or not a net increase in diffusion rate occurs depends partially on the competition between these processes. In Fig. 8 (middle), for $2.1 \mathrm{MeV}$ electrons the diffusion rate curves are coincident for all five chosen wave distributions. This is because the region of resonance for $2.1 \mathrm{MeV}$ electrons is approximately $|\lambda|<5^{\circ}$. However, for $10 \mathrm{MeV}$ electrons, whose resonance region extends to $|\lambda| \sim 30^{\circ}$, the diffusion rates increase substantially as the latitudinal range of the wave distribution increases. The differences and similarities between Fig. 7 and Fig. 8 regarding the influence on the diffusion rates of the density distributions and the wave distributions are largely controlled by the latitudinal regions of resonant electron-wave interaction.

In the bottom panel of Fig. 8 we show a two-dimensional plot of the bounce-averaged pitch-angle diffusion rate for EMIC waves at $L=4$, for Wave Distribution $|\lambda|<20^{\circ}$ and density model 2 . The bottom panels of Fig. 7 and Fig. 8 provide a ready comparison between the scattering properties of hiss and EMIC waves for the adopted wave and density distributions.

In the present study we consider electron interaction with EMIC waves in a hydrogen plasma only. Electron scattering by EMIC waves can, in fact, be sensitively dependent on the ion composition of the plasma (Summers and Thorne, 2003; Summers et al., 2007b). The influence of latitudinal distributions of particle density and wave power on electron scattering by EMIC waves in a multi-ion $\left(\mathrm{H}^{+}, \mathrm{He}^{+}, \mathrm{O}^{+}\right)$ plasma is left as an interesting topic for future study.

\subsection{Electron loss timescales due to combined scatter- ing by VLF chorus, ELF hiss and EMIC waves}

We now consider electron precipitation loss timescales due to combined scattering by VLF chorus, ELF hiss and EMIC waves. In Fig. 9 (Case (I)) we show a schematic representation of the plasmasphere and typical distribution of waves for the case of low geomagnetic activity. Figure 9 (Case (II)) depicts the plasmasphere incorporating two drainage plumes, together with an expected distribution of waves. The plasmaspheric configurations in Fig. 9 were constructed from profiles presented by Spasojevic et al. (2003) that were deduced from IMAGE satellite data (see Summers et al. (2007b), section 5, for other examples of observed global configurations of the plasmasphere). The tables in Fig. 9 show the approximate percentage of an electron (circular) drift orbit that traverses each wave mode for $L=3,4,5,6,7$. In Fig. 10 we present the total electron loss timescales, at energies $100 \mathrm{keV}, 300 \mathrm{keV}, 1 \mathrm{MeV}$, $3 \mathrm{MeV}$, due to combined scattering by the chorus, plasmaspheric hiss and EMIC wave distributions depicted in Cases (I) and (II) in Fig. 9. It is assumed that inside the plasmasphere (/plumes) the particle number density satisfies 

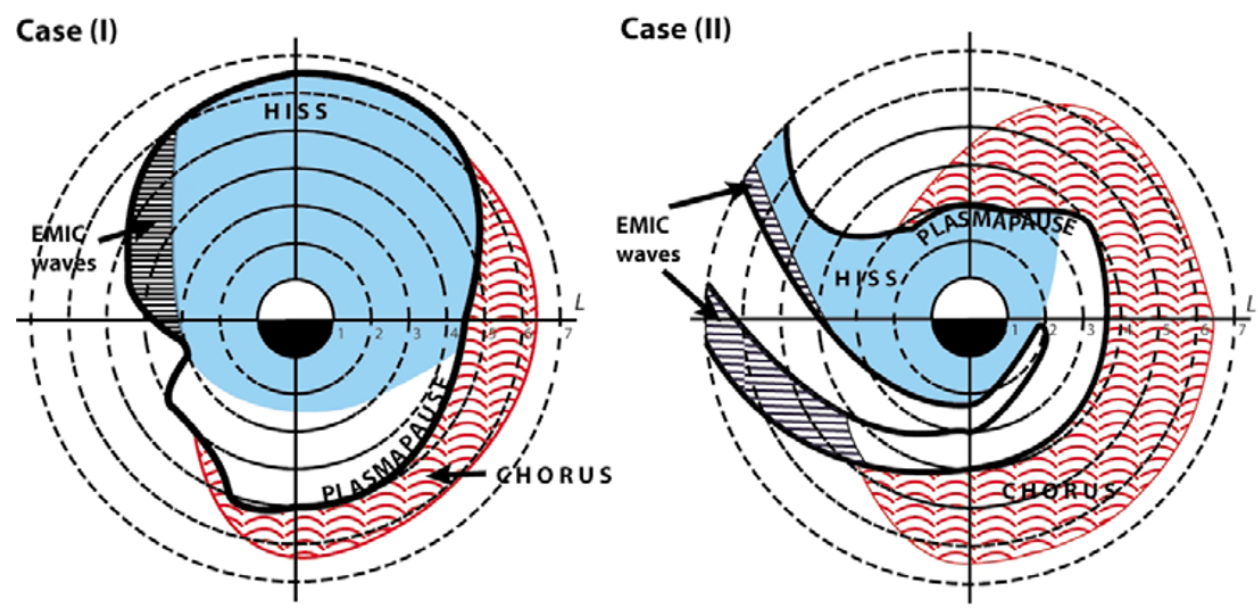

DRIFT-AVERAGE \% FOR EACH WAVE MODE

\begin{tabular}{|c|c|c|c|c|c|}
\hline & $\mathrm{L}=3$ & 4 & 5 & 6 & 7 \\
\hline chorus & 0 & 0 & $32 \%$ & $39 \%$ & 0 \\
\hline hiss & $66 \%$ & $45 \%$ & $32 \%$ & $23 \%$ & 0 \\
\hline $\begin{array}{c}\text { EMIC } \\
\text { waves }\end{array}$ & 0 & $9 \%$ & $6 \%$ & 0 & 0 \\
\hline
\end{tabular}

\begin{tabular}{|c|c|c|c|c|c|}
\hline & $\mathrm{L}=3$ & 4 & 5 & 6 & 7 \\
\hline chorus & $17 \%$ & $53 \%$ & $58 \%$ & $49 \%$ & 0 \\
\hline hiss & $30 \%$ & $8 \%$ & $4 \%$ & $3 \%$ & $3 \%$ \\
\hline $\begin{array}{l}\text { EMIC } \\
\text { waves }\end{array}$ & 0 & $1 \%$ & $10 \%$ & $8 \%$ & $5 \%$ \\
\hline
\end{tabular}

Fig. 9. Schematic distributions of chorus, plasmaspheric hiss and EMIC waves in the case of low geomagnetic activity and expanded plasmasphere (Case (I)) and following high geomagnetic activity during the evolution of plasmaspheric plumes (Case (II)). For each case, the corresponding table shows the approximate percentage of an electron drift orbit that traverses each wave mode for a given $L$-value.

Table 1. The three wave models adopted in association with Figs. 9 and 10.

\begin{tabular}{llcc}
\hline & Wave Model (a) & Wave Model (b) & Wave Model (c) \\
\hline Chorus & $\Delta B(\lambda)=100 \mathrm{pT}$ & Wave Model 4 & Wave Model 5 \\
Hiss & $\Delta B(\lambda)=100 \mathrm{pT}$ & Wave Model III & $\Delta B(\lambda)=10 \mathrm{pT}$ \\
EMIC & $\Delta B(\lambda)=1 \mathrm{nT}$ & $\Delta B(\lambda)=1 \mathrm{nT}$ & $\Delta B(\lambda)=1 \mathrm{nT}$ \\
\hline
\end{tabular}

$N(\lambda)=N_{\text {eq }}[f(\lambda)]^{\sigma}, N_{\text {eq }}=1390(3 / L)^{4.83} \mathrm{~cm}^{-3}$, and that outside the plasmasphere (/plumes) $N(\lambda)=N_{\text {eq }}[f(\lambda)]^{\kappa}$, $N_{\text {eq }}=124(3 / L)^{4} \mathrm{~cm}^{-3}$. We adopt three sets of latitudinal distributions of wave power, namely, Wave Models (a), (b), (c) as specified in Table 1. We further assume that the chorus, hiss and EMIC waves have Gaussian spectral densities with the same parameters as used in the respective Figs. 1, 7 , and 8 . We calculate the total electron loss timescale $\tau_{\text {tot }}$ using the method described by Summers et al. (2007b) (section 5). The method involves expressing the total electron scattering rate as the sum of the scattering rates due to each wave mode, each rate being evaluated at the equatorial loss cone angle. We estimate electron loss timescales by using the scattering rate at the edge of the loss cone only in those cases in which the pitch-angle diffusion rate is small over a high pitch-angle range narrower than $75^{\circ}<\alpha_{\text {eq }}<90^{\circ}$. Use of this criterion should ensure that electron lifetimes are controlled by scattering rates near the edge of the loss cone (e.g., Shprits et al., 2006). In Fig. 10 the influence on the total electron loss timescale $\tau_{\text {tot }}$ (which is inversely proportional to the total scattering rate) of the latitudinal distributions of density and wave power in general reflects the results obtained in Figs. 1, 2, 4, 7, and 8. For example, with some exceptions, the loss timescale increases as the latitudi- nal density distribution becomes stronger, or the latitudinal wave distribution becomes weaker, or the electron energy increases. A key factor controlling the results in Fig. 10 is that for a particular wave mode to contribute to the total scattering rate at a given $L$-value, an electron must not only traverse a region containing that wave mode, but the electron kinetic energy must exceed the minimum energy for resonance. In this regard, it is interesting to note the influence of EMIC waves in Case (I) and Case (II) in Fig. 10. In both Case (I) and Case (II) EMIC waves do not influence the loss timescales for $100 \mathrm{keV}, 300 \mathrm{keV}$, and $1 \mathrm{MeV}$ electrons since these energies are below the minimum resonant energy for electron-EMIC wave interaction. However, in Case (I) for $4<L<5$ and in Case (II) for $4<L<7$, EMIC waves resonantly scatter $3 \mathrm{MeV}$ electrons with the result that since these waves are strong $(\Delta B=1 \mathrm{nT})$ the total electron loss timescale is significantly reduced. In Case (II) for $3 \mathrm{MeV}$ electrons in the range $4<L<7$ since electron scattering is dominated by EMIC waves, and since also the region for EMIC wave-electron resonance is close to the equator, an increase in particle density at higher latitude has only marginal effect on the total loss timescale. 


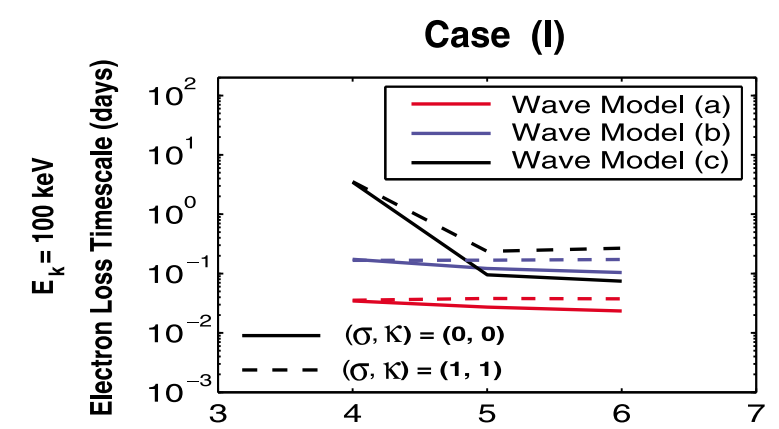

Case (II)
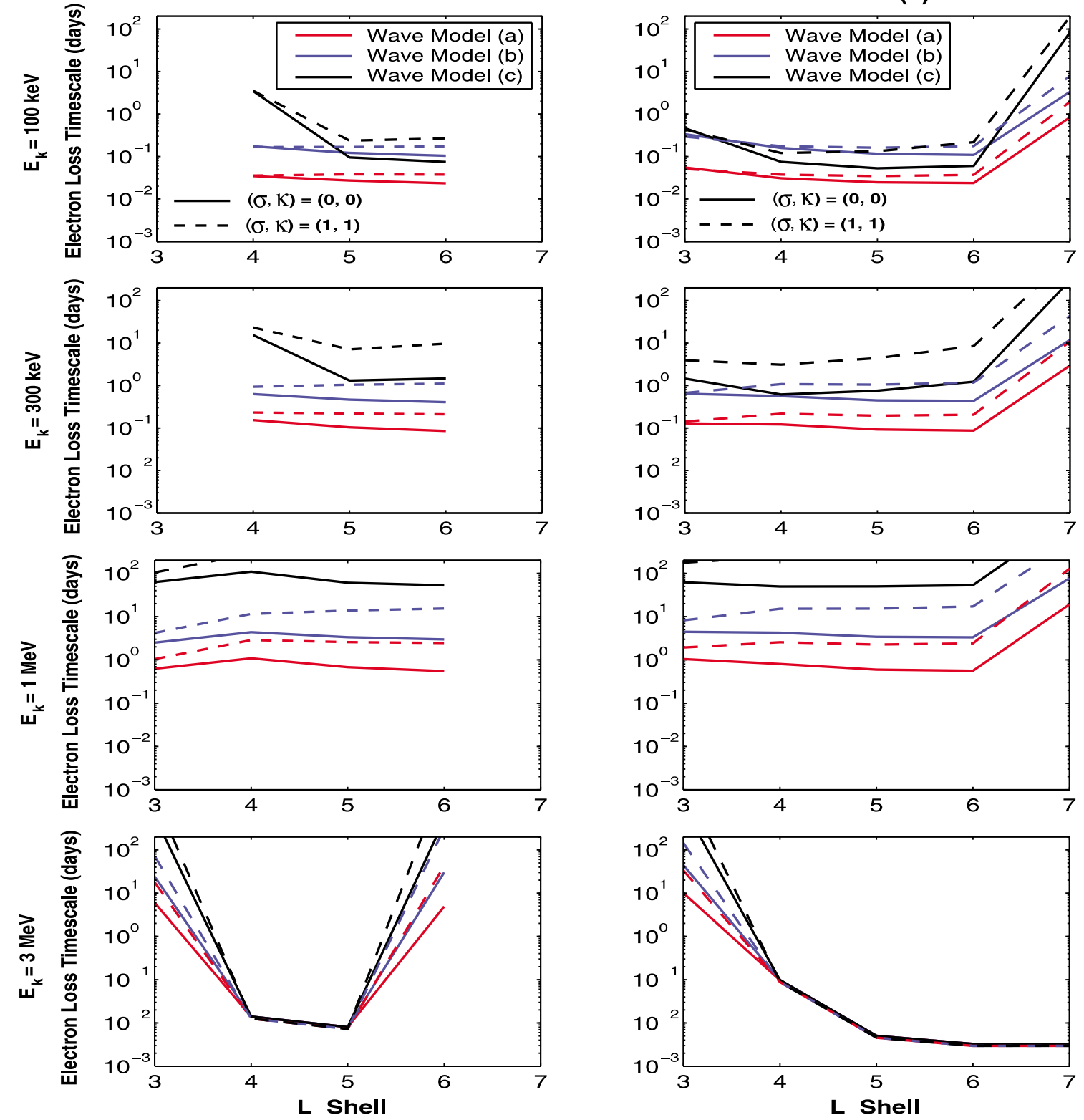

Fig. 10. Corresponding to Fig. 9, total electron loss timescales due to combined scattering by chorus, plasmaspheric hiss and EMIC waves in Case (I) and Case (II). The wave models (a), (b), (c) are given in Table 1 and the particle density models specified by $\sigma$ and $\kappa$ are described in the text.

\section{Summary}

We have examined the cyclotron resonant bounceaveraged diffusion rates of radiation belt electrons for VLF chorus, ELF hiss and EMIC waves, and we have adopted various latitudinal distributions of particle density and wave power. The diffusion rates can depend sensitively on the specified distributions of density and wave power. In general, however, the extent to which the distributions of density and wave power influence the diffusion rates depends on wave mode, equatorial pitch-angle, electron energy, and $L$-value. The bounce-averaged diffusion rates fundamentally depend on the spatial region over which cyclotron resonant wave-particle interaction can take place for any particular wave mode. The regions for resonance depend on wave band, electron energy, equatorial pitch-angle, and the local values of particle number density and magnetic field. Accurate determination of the cyclotron resonant diffusion rates of radiation belt electrons clearly requires detailed data on the global distribution of particle density and wave power. This study confirms the need for the acquisition of such data from future satellite missions.

Acknowledgments. This work is supported by the Natural Sciences and Engineering Research Council of Canada under grant A-0621.

\section{References}

Albert, J. M., Evaluation of quasi-linear diffusion coefficients for EMIC waves in a multispecies plasma, J. Geophys. Res., 108(A6), 1249, doi: 10.1029/2002JA009792, 2003.

Albert, J. M., Simple approximations of quasi-linear diffusion coefficients, J. Geophys. Res., 112, A12202, doi:10.1029/2007JA012551, 2007.

Denton, R. E., et al., Distribution of density along magnetospheric field lines, J. Geophys. Res., 111, A04213, doi:10.1029/2005JA011414, 2006.

Fraser, B. J. and T. S. Nguyen, Is the plasmapause a preferred source 
region of electromagnetic ion cyclotron waves in the magnetosphere?, J. Atmos. Sol. Terr. Phys., 63, 1225-1247, 2001.

Horne, R. B., et al., Timescale for radiation belt electron acceleration by whistler mode chorus waves, J. Geophys. Res., 110, A03225, doi:10.1029/2004JA010811, 2005.

Lyons, L. R., Pitch angle and energy diffusion coefficients from resonant interactions with ion-cyclotron and whistler waves, J. Plasma Phys., 12, 417-432, 1974.

Meredith, N. P., R. B. Horne, and R. R. Anderson, Substorm dependence of chorus amplitudes: Implications for the acceleration of electrons to relativistic energies, J. Geophys. Res., 106, 13165-13178, 2001.

Meredith, N. P., R. B. Horne, R. M. Thorne, D. Summers, and R. R. Anderson, Substorm dependence of plasmaspheric hiss, J. Geophys. Res., 109, A06209, doi:10.1029/2004JA010387, 2004.

Miyoshi, Y., et al., Rebuilding process of the outer radiation belt during the 3 November 1993 magnetic storm: NOAA and Exos-D observations, $J$. Geophys. Res., 108(A1), 1004, doi:10.1029/2001JA007542, 2003.

Omura, Y. and D. Summers, Dynamics of high-energy electrons interacting with whistler mode chorus emissions in the magnetosphere, J. Geophys. Res., 111, A09222, doi:10.1029/2006JA011600, 2006.

Reinisch, B. W., et al., Plasma density distribution along the magnetospheric field: RPI observations from IMAGE, Geophys. Res. Lett., 28(24), 4521-4524, 2001.

Roth, I., M. Temerin, and M. K. Hudson, Resonant enhancement of relativistic electron fluxes during geomagnetically active periods, Ann. Geophys., 17, 631-638, 1999.

Santolik, O., D. A. Gurnett, J. S. Pickett, M. Parrot, and N. Cornilleau-Wehrlin, A microscopic and nanoscopic view of stormtime chorus on 31 March 2001, Geophys. Res. Lett., 31, L02801, doi:10.1029/2003GL018757, 2004.

Sheeley, B. W., M. B. Moldwin, H. K. Rassoul, and R. R. Anderson, An empirical plasmasphere and trough density model: CRRES observations, J. Geophys. Res., 106, 25631-25642, 2001.

Shprits, Y. Y., W. Li, and R. M. Thorne, Controlling effect of the pitch angle scattering rates near the edge of the loss cone on electron lifetimes, J. Geophys. Res., 111, A12206, doi:10.1029/2006JA011758, 2006.
Spasojevic, M., et al., Global response of the plasmasphere to a geomagnetic disturbance, J. Geophys. Res., 108(A9), 1340, doi:10.1029/ 2003JA009987, 2003.

Summers, D., Quasi-linear diffusion coefficients for field-aligned electromagnetic waves with applications to the magnetosphere, J. Geophys. Res., 110, A08213, doi:10.1029/2005JA011159, 2005.

Summers, D. and C. Ma, A model for generating relativistic electrons in the Earth's inner magnetosphere based on gyroresonant wave-particle interactions, J. Geophys. Res., 105, 2625-2640, 2000.

Summers, D. and R. M. Thorne, Relativistic electron pitch angle scattering by electromagnetic ion cyclotron waves during geomagnetic storms, $J$. Geophys. Res., 108(A4), 1143, doi:10.1029/2002JA009489, 2003.

Summers, D., R. M. Thorne, and F. Xiao, Relativistic theory of waveparticle resonant diffusion with application to electron acceleration in the magnetosphere, J. Geophys. Res., 103, 20487-20500, 1998.

Summers, D., et al., Model of the energization of outer-zone electrons by whistler-mode chorus during the October 9, 1990 geomagnetic storm, Geophys. Res. Lett., 29(24), 2174, doi:10.1029/2002GL016039, 2002.

Summers, D., B. Ni, and N. P. Meredith, Timescales for radiation belt electron acceleration and loss due to resonant wave-particle interactions: 1. Theory, J. Geophys. Res., 112, A04206, doi:10.1029/2006JA011801, 2007a.

Summers, D., B. Ni, and N. P. Meredith, Timescales for radiation belt electron acceleration and loss due to resonant wave-particle interactions: 2 . Evaluation for VLF chorus, ELF hiss, and electromagnetic ion cyclotron waves, J. Geophys. Res., 112, A04207, doi:10.1029/2006JA011993, 2007 b.

Thorne, R. M., T. P. O'Brien, Y. Y. Shprits, D. Summers, and R. B. Horne, Timescale for MeV electron microburst loss during geomagnetic storms, J. Geophys. Res., 110, A09202, doi:10.1029/2004JA010882, 2005.

D. Summers (e-mail: dsummers@math.mun.ca) and B. Ni 